\title{
ESTRUCTURACIÓN DE BUENAS PRÁCTICAS AGRÍCOLAS Y APLICACIÓN DE LA RADIACIÓN UV-C EN TOMATE DE ÁRBOL (Solanum betaceum Cav.).
}

\author{
Autores: \\ Jorge Viteri ${ }^{1}$ \\ Marta Cruz ${ }^{1}$ \\ Juan Bravo' \\ Fausto Viteri'
}

Facultad de Ciencias de la Ingeniería, Universidad Tecnológica Equinoccial, Av. Occidental y Av. Mariana de Jesús, Apartado 17-01-2764, Quito - Ecuador jviteri@ute.edu.ec - bvje2633@ute.edu.ec - vmfr2366@ute.edu.ec 



\section{Resumen}

En los últimos años ha aumentado significativamente la demanda y comercio de frutas exóticas, a nivel mundial. Este incremento ocasiona que en los países de origen se implementen procedimientos y apliquen normas de calidad y fitosanitarias al cultivo mismo y al manejo poscosecha del producto en fresco de manera que cumplan con los requisitos fitosanitarios y de calidad.

Para impulsar la actividad exportadora de alto valor agregado del sector agrícola se realizó un estudio y análisis de las Buenas Prácticas Agrícolas (BPA) del cultivo de Tomate de Árbol (Solanum betaceum Cav.), en conjunto con los distintos actores que componen la cadena agroalimentaria.

Estas BPA se detallaron en una Guía de Buenas Prácticas Agrícolas y se recopiló en un afiche divulgativo para una fácil comprensión de los agricultores.

Se determinó los principales problemas poscosecha del tomate de árbol y se desarrolló una posible solución con la aplicación de la tecnología de radiación de luz ultra violeta (UV). Esta tecnología permitió disminuir el daño por contaminación microbiológica extendiendo su vida útil por 45 días.

PALABRAS CLAVE: Tomate de árbol, BPA, Radiación UV

\section{Abstract}

In recent years has significantly increased demand and global trade of exotic fruits, in turn this increased demand has caused in countries of origin procedures are implemented and enforced standards of quality and phytosanitary the same culture and postharvest handling of fresh produce so that they know the requirements of phytosanitary and quality.

To boost the export performance of high value-added agricultural sector, a study and analysis of Good Agricultural Practice (GAP) of the tree tomato crop, in conjunction with the various actors that make up the food chain.

These GAP were detailed in a written document called the Guide to Good Agricultural Practices, and collected in a poster for easy understanding of farmers.

It identified major postharvest problems in tree tomato, and developed a possible solution to the application of radiation technology for ultra violet light (UV). This technology helped to reduce the damage by microbiological contamination so that life was extended for 45 days.

KEY WORDS: Tree tomato, GAP, Radiation UV

Recibido: Mayo 2011

Aprobado: Julio 2011 


\section{INTRODUCCIÓN}

En los últimos años ha aumentado significativamente la demanda y comercio de frutas exóticas, a nivel mundial. Este incremento en la demanda ha ocasionado que en los países de origen se implementen procedimientos y se apliquen normas de calidad y fitosanitarias al cultivo mismo y al manejo poscosecha del producto fresco de manera que cumpla con los requisitos fitosanitarios y de calidad.

Su importancia radica en que la implementación de las Buenas Prácticas Agrícolas (BPA) no sólo garantiza que los alimentos sean aptos para el consumo humano sino, que permite acceder a distintos mercados que en sus legislaciones las incluyen. Consecuentemente el productor que aplica BPA puede colocar sus productos en mercados externos cada vez más exigentes y competitivos así como también diferenciar el producto en el mercado interno.

En el Ecuador existen agricultores dedicados al cultivo de tomate de árbol, pero muy pocos disponen de oportunidades de exportar. Esto es consecuencia de varios factores entre los que se pueden enumerar: la baja productividad de sus cultivos, poca calidad del fruto cosechado, pérdidas poscosecha del tomate, reducida extensión de terreno dedicado a este cultivo, así como el escaso acceso a los recursos económicos y tecnológicos que permitan optimizar su producción.

El tomate de árbol (Solanum tuberosum Cav) corresponde al tipo biológico de arbusto semileñoso; alcanza 2 ó 3 metros de altura y presenta ciclo vegetativo perenne. Crece en zonas con altitudes que varían de 1000 a 3000 msnm. En altitudes inferiores a 1000 msnm no fructifica bien porque durante la noche la temperatura no es lo suficientemente baja.

Es una planta de climas templados y fríos. Su temperatura está entre 13 a $24^{\circ} \mathrm{C}$ siendo la óptima entre 16 y $19^{\circ} \mathrm{C}$. No necesita gran humedad atmosférica, razón por la cual, se cultiva frecuentemente en zonas altas de clima seco.

La producción empieza al año y medio o dos años después de la siembra, siendo intensa solamente por 4 ó 5 años ( 5 meses /año) y puede durar de 10 a 12 años.

Los frutos son bayas ovoides, cuyo mesocarpio (pulpa) es de color amarillo, rosado o rojo; ésta es la parte utilizable. Las variedades con frutos que presentan pulpa rojo oscura y semillas negras, se prefieren a los de pulpa rosada y semillas claras.

La sierra ecuatoriana posee varias zonas óptimas para la producción de esta fruta; zonas caracterizadas por un clima templado y fresco, y suelos con buen contenido de materia orgánica. Las provincias más representativas en cultivos de esta fruta son Imbabura, Tungurahua y Pichincha.

Las variedades de tomate de árbol que se producen en el Ecuador son el redondo y el gigante morado y anaranjado.

Para el almacenamiento, durante el periodo de poscosecha del tomate de árbol según Portela (1999), las condiciones óptimas son de 3 a $4.5{ }^{\circ} \mathrm{C}$ y de $90-95 \%$ de humedad relativa, ya que por debajo de $3^{\circ} \mathrm{C}$ los frutos sufren el deterioro conocido como daño por frío (pardeamiento de la piel y presencia de pequeñas depresiones en la superficie del fruto). En cambio a temperaturas mayores que $4.5{ }^{\circ} \mathrm{C}$ las pérdidas por podredumbres (hongos) aumentan marcadamente.

El hongo no necesita heridas para penetrar el fruto. Normalmente los hongos infectan la fruta cuando está en el árbol pero no se desarrolla la enfermedad sino hasta el almacenamiento. Un baño de 8-10 minutos en agua a $50^{\circ} \mathrm{C}$ inmediatamente después de la cosecha controla efectivamente las infecciones latentes, pero causa daño en el pedúnculo y en la capa de cera de la cutícula de los 
frutos, aumentando la pérdida de agua durante el almacenamiento. (Portela, 1999)

Una alternativa para controlar el daño por microorganismos durante la poscosecha del tomate de árbol, es la aplicación de la radiación Ultravioleta espectro C (UV-C) a los frutos inmediatamente después a la cosecha.

La radiación UV-C es la exposición a la luz ultravioleta (longitud de onda entre 200 y $290 \mathrm{~nm}$ ) de las frutas con el objetivo de reducir la carga microbiana inicial en la superficie del producto. Esta tecnología forma parte de las llamadas "tecnologías limpias", que permite prolongar la vida útil y la calidad de frutas y hortalizas sin alterar sus características nutritivas, físicas y organolépticas. Por ejemplo se observó que tratamientos de luz UV-C redujo la incidencia y la severidad del daño por frío en el ají chile campana, lo cual fue evidenciado a nivel metabólico por reducción en la fuga de electrolitos de las paredes celulares, tasa de respiración y contenido de fenoles, también ha observado efectos beneficiosos de la UV-C para reducir el daño por frío en mangos (Von de Becke, 1999; Fonseca, 2009; González-Aguilar y col., 2001).

El presente trabajo tiene como objetivo principal, estudiar los problemas postcosecha de tomate de árbol (Solanum betaceum Cav.) y determinar una estrategia de Buenas Prácticas Agrícolas (BPA).

\section{METODOLOGÍA}

El trabajo de investigación se dividió en las siguientes etapas:

- Determinación de los principales problemas poscosecha del tomate de árbol y diseño de una estrategia de Buenas Prácticas Agrícolas (BPA).

- Estudio de la aplicación de radiación UV-C como tratamiento poscosecha.

\section{Determinación de problemas poscosecha del tomate de árbol}

Para la ejecución de este proyecto se realizaron actividades en conjunto con los siguientes actores: Agricultores de tomate de árbol de la sierra centro del Ecuador, Municipio de Pelileo, Instituto de Investigación Agropecuaria (INIAP) y la Planta procesadora Hortifrutícola Ambato C.A. (PLANHOFA). Estas actividades estuvieron encaminadas a concretar el papel de cada uno de los actores dentro de la cadena agroindustrial del tomate de árbol.

Los problemas de poscosecha del tomate de árbol se identificaron por medio de visitas técnicas a los agricultores, así como de la revisión bibliográfica del tema.

\section{Estudio de la aplicación de radiación UV-C como tratamiento poscosecha}

La aplicación de la radiación UV-C en tomates de árbol se realizó de la siguiente manera: los frutos fueron cosechados e inmediatamente trasladados al laboratorio en la Facultad de Ciencias de la Ingeniería, en donde se seleccionaron por diferentes atributos como la apariencia, color uniforme y ausencia de defectos (Figura 1). Con el fin de eliminar todo tipo de cuerpos extraños que pudieran traer los frutos se lavaron con agua potable, se desinfectaron con una solución de 250 ppm de hipoclorito; lavados y secos los frutos se dividieron en dos grupos: uno para el tratamiento con UV-C y el otro para control. La energía aplicada fue de $13 \mathrm{~kJ} / \mathrm{m}^{2}$.

Se colocaron $500 \mathrm{~g}$ de fruta en bandejas de poliestireno espumado (Largo: $210 \mathrm{~m} /$ Ancho: $145 \mathrm{~mm}$ / Altura: $20 \mathrm{~mm}$ ) y se cubrieron con una película de polietileno estirable de 20 micras de espesor. Todas las frutas se almacenaron a $7^{\circ} \mathrm{C}$ por 48 días. 


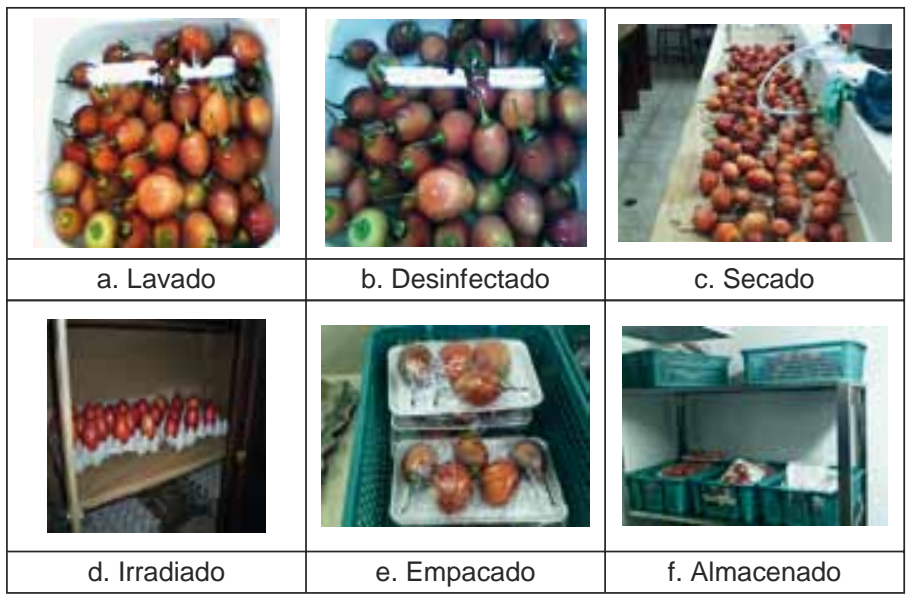

Figura 1. Procedimiento de aplicación de la radiación de UV-C en tomate de árbol.

Se evaluó el efecto de radiación UV-C sobre el crecimiento de microorganismos tales como el recuento de aerobios mesófilos totales, mohos y levaduras a los 0, 12, 24, 36 y 48 días. Todos los análisis se realizaron por triplicado.

Para el recuento de levaduras y mohos las placas se incubaron según la Guía de interpretación 3M Petrifilm la cual sugiere incubarlas durante $3-5$ días a $25^{\circ} \mathrm{C}$. Mientras que para el recuento de microorganismos aerobios mesófilos totales las placas se incubaron durante 48 horas a $35^{\circ} \mathrm{C}$.

\section{RESULTADOS Y DISCUSIÓN}

\section{Guía de Buenas Prácticas Agrícolas (BPA)}

Los principales problemas poscosecha del tomate de árbol son consecuencia de un incorrecto manejo en la parte agrícola y de la cosecha; y al inadecuado control de empacado, almacenamiento y transporte.

En el empacado, las prácticas que se utilizan son muy precarias, ya que se emplean cajas de madera de aproximadamente $15 \mathrm{~kg}$, en donde no se aplica procedimientos adecuados de manipulación del fruto.

Otro de los problemas encontrados durante la poscosecha y que requiere una enorme atención, es consecuencia del maltrato que se da a las cajas durante el transporte y es causa de grandes pérdidas en la calidad del fruto.

El daño llamado antracnosis causada por el hongo Colletotrichum gloeosporioides presenta una lesión profunda en el fruto como se indica en la figura 2.

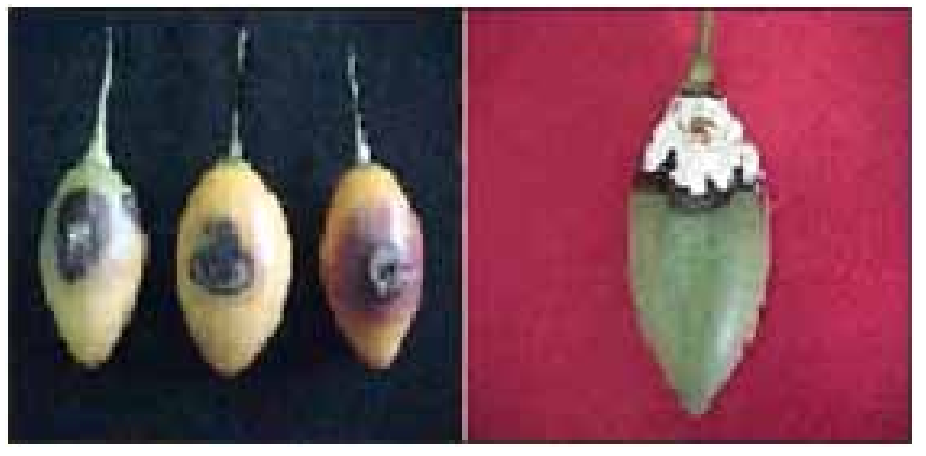

Figura 2. Tomate de árbol con antracnosis.

Para mejorar el manejo agrícola se elaboró la guía de Buenas Prácticas Agrícolas, que para su estructura se consideró los siguientes aspectos:

- Material vegetal de propagación

- Historial y manejo del suelo

- Gestión del suelo y de los sustratos

- Fertilización

- Protección de cultivos

En cada uno de los aspectos se detalla la forma idónea de mantener un cultivo sostenible, sustentable y con mayor índice de productividad, para así obtener tomates de árbol con calidad de exportación. 
Esta guía de Buenas Prácticas Agrícolas se recopila en una hoja divulgativa, de fácil comprensión por los agricultores y otros actores (figura 3).



Figura 3. Guía de Buenas Prácticas Agrícolas del Tomate de árbol.

\section{Estudio de la aplicación de radiación Ultravioleta para disminuir pérdidas en poscosecha en el tomate de árbol}

Los resultados de los análisis microbiológicos durante los tiempos de almacenamiento se presentan en la figura 4. Se observa que el crecimiento de microorganismos de aerobios totales (a), mohos (b) y levaduras (c) se ha retardado considerablemente en el tómate de árbol sometido a radiación UV-C. Con respecto a los aerobios en las dos muestras, tomates tratados y control, no existe presencia a los 48 días, porque además del efecto de la radiación, el incremento de levaduras inhibe su crecimiento.

Para las levaduras el tratamiento con UV-C muestra una efectividad del $41.6 \%$ a diferencia del control.

La efectividad de la irradiación en los mohos fue del 100\% en los tomates que estuvieron expuestos al tratamiento con UV-C, a diferencia de los controles que si presentaron presencia de mohos a los 48 días.

El tómate de árbol no presentó cambios organolépticos después del uso de radiación UV-C ni al finalizar el tiempo del estudio de 48 días.

La aplicación de la radiación UV-C a tomates de árbol permitió que la fruta no presente crecimiento de microorganismos en su corteza, lo que disminuye la presencia de antracnosis. Este beneficio se refleja en un incremento de vida útil del tomate de árbol disminuyendo las pérdidas poscosecha.

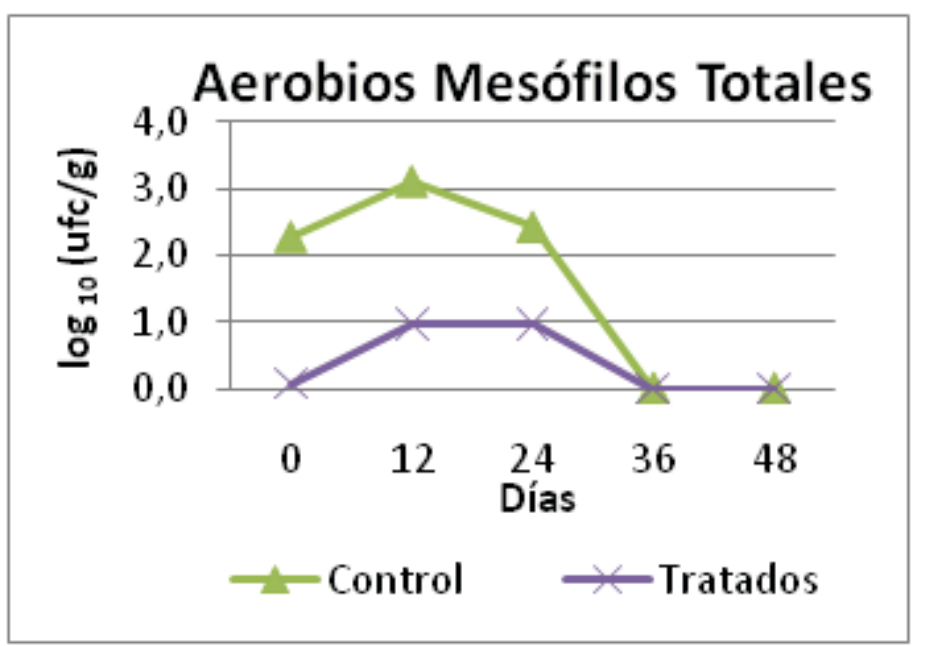

a) Recuento de aerobios mesófilos 


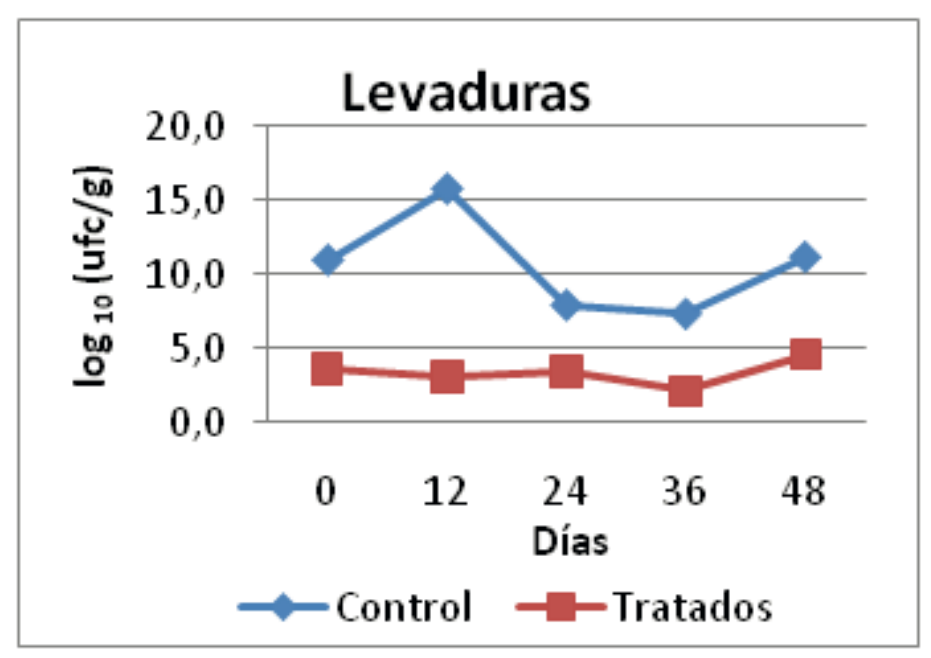

b) Recuento de levaduras

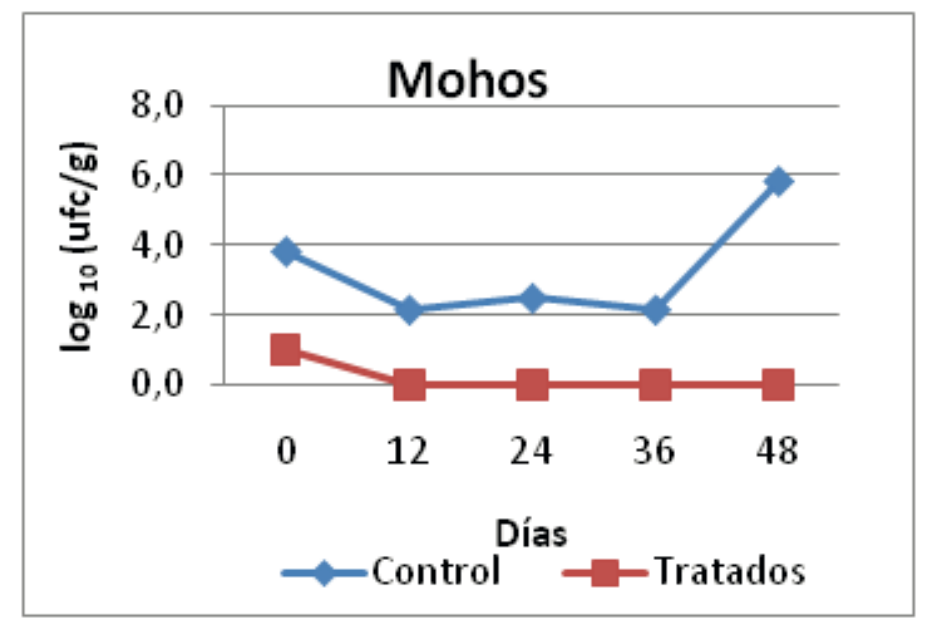

c) Recuento de mohos.

Figura 4. Curvas del crecimiento microbiano en los tomates de árbol.

\section{CONCLUSIONES}

Para un adecuado manejo agrícola de la fruta se elaboró una guía de Buenas Prácticas Agrícolas que permitirá obtener frutos con buena calidad.
Al final de los 48 días la aplicación de la dosis de $13 \mathrm{~kJ} / \mathrm{m}^{2}$ de radiación UV-C en el tomate de árbol permitió reducir entre 40 y $100 \%$ el desarrollo de microorganismos aerobios mesófilos, mohos y levaduras.

La preservación de la fruta mediante irradiación UV-C puede prolongar la vida en percha en un máximo de 48 días, lo que permitirá mejorar los tiempos de exportación para enviar, mediante transportes más accesibles como barcos, a los mercados internacionales.

La formulación de las Buenas Prácticas Agrícolas específicas para el tomate de árbol, posteriormente va a servir para mejorar la producción de la fruta para que se pueda acceder a mejores mercados mundiales.

Los productores de la zona centro de la serranía ecuatoriana tienen la posibilidad de mejorar sus prácticas agrícolas, y de esta manera incrementar las oportunidades de colocar al tomate árbol en el mercado internacional.

La aplicación de este proyecto contribuirá a la construcción del buen vivir de la población mediante el fortalecimiento de la seguridad alimentaria del país a través de la generación de ingresos por acceso a mercados, la protección al medio ambiente mediante la producción sustentable y mejora las condiciones laborales de los productores de tomate de árbol de la zona centro del Ecuador y de sus familias.

\section{AGRADECIMIENTOS}

Este trabajo fue financiado por la Universidad Tecnológica Equinoccial dentro del Concurso interno de Proyectos de Investigación. 


\section{BIBLIOGRAFÍA}

BCE (Banco Central del Ecuador). 2009. Comercio Exterior: Consulta de Totales por Nandina - País (en línea). Quito, EC. Consultado 26 jul. 2009. Disponible en http://www.portal.bce.fin.ec/vto_ bueno/seguridad/ComercioEx teriorEst.jsp.

Cadena, Esteban. 2002. Estudio de Prefactibilidad de Tomate de árbol. SICA.

Caicedo, Bolaños, Cruz. 2008. Estudio de las posibilidades agroindustriales del tomate de árbol (Solanum betaceum Cav.). No. 1.

MAG. Ecuador. Comercialización del tomate de árbol. Coordinadora ecuatoriana de agroecología. Recuperado el junio 2010. En: http://www.ceaecuador.org/ imagesFTP/ 4791.tomate_arbol_ mag_comercializacion.pdf

Convenio MAG/IICA (Ministerio de Agricultura y Ganadería/Instituto Interamericano de Cooperación para la Agricultura): Subprograma de Cooperación Técnica. 2001. Identificación de mercados y tecnología para productos agrícolas tradicionales de exportación: Tomate de Árbol/Tamarillo/TreeTomato/ SweetTomato. Quito, Ecuador. Consultado 14 jul. 2009. En: http://www.sica.gov.ec/agronegocios/biblioteca/Convenio\%20 MAG\%20IICA/ productos/tomate_arbol_mag.pdf.

Corpoica. 2003. Tecnología para el cultivo del tomate de árbol. Medellín - Colombia.

Fonseca, J. 2009. Efectos de la luz UV-C en calidad de hortalizas. Fecha de consulta: 2010-05-12. En: http://www.hortalizas.com/ pdhca/?storyid=1604.

Gil, P. 2008. Estado actual del tomate de árbol en el mercado español (entrevista) Barcelona, ES. Mercabarna.

Global G.AP. 2007. Puntos de control y criterios de cumplimiento para aseguramiento integrado de fincas, módulo para frutas y hortalizas.
González-Aguilar, G.A.; Wang, C. y Buta, G. (2004). UV-C irradiation reduces breakdown and chilling injury of peaches during cold storage. J. Sci. Food Agric. 84: 415-442.

Guerrero-Beltrán, J.A. Barbosa-Cánovas, G.V. 2004. Review: Advantages and Limitations on Processing Foods by UV Light. Food Science Technology International. EUA.

INIAP. 2004. Manual del Cultivo de tomate de árbol. Quito - Ecuador.

León Juan, Viteri Pablo, Cevallos Guillermo. 2004. Manual del Cultivo de Tomate de Árbol. Estación Experimental Santa Catalina. Programa de Fruticultura Granja Experimental Tumbaco.

León, Juan, 1996. Guía para el cultivo de Tomate de árbol, INIAPCOTESU.

MAG e IICA, 2001. Identificación de Mercados y Tecnologías para Productos Andinos Tradicionales de Exportación. Tomate de Árbol. Convenio MAG/IICA Subprograma de Cooperación Técnica. Quito - Ecuador.

Pacheco. C. 2008. Los frutales tienen demanda foránea (en línea) Quito, EC. Consultado 14 jul. 2009. Disponible en http://www. hoy.com.ec/noticias-ecuador/los-frutales-tienen-demandaforanea-301936-301936.html.

Parkin, M. 2006. Microeconomía 7ed. Versión para Latinoamérica. Editorial Addison-Wesley. Argentina.

Portela, S. I. 1999. Fisiología y manejo de postcosecha del tamarillo (Cyphomandra betacea). Department of Vegetable Crops.

Proexant (Promoción de Exportaciones Agrícolas no Tradicionales). 2007. Estudio de Mercado para Tomate de Árbol (Cyphomandra betacea), Uchuva (Physalis peruviana I.), y Granadilla (Passiflora Ligularis), en Ecuador, la Unión Europea y Asia, como frutas frescas y procesadas. Desarrollo tecnológico para el mejoramiento del manejo post-cosecha de frutales exportables de interés para los países andinos. No. 6: 10 . 
SESA, 2006. Guía de Buenas Prácticas Agrícolas.

SICA (Servicio de Información del Censo Agropecuario). 2001. Estudio de pre factibilidad para tomate de árbol (en línea). Quito, EC. Consultado 14 jul. 2009. En http://www.sica.gov.ec/ agronegocios/productos $\% 2$

Van der Vecke C.H. 1999. Radiación Ultravioleta. Glosario de términos, artículos y textos. Fecha de consulta: 10/02/10. En: http// members.fortunecity.es/ rednovohcop/radiacuv.html

Wikipedia. 2009. Unión Europea (en línea). Madrid, ES. Consultado 14 jul. 2009. En http://es.wikipedia.org/wiki/Uni\%C3\%B3n_ Europea. 